\title{
Preclinical Evidence of Curcuma longa and Its Noncurcuminoid Constituents against Hepatobiliary Diseases: A Review
}

\author{
Soyeon An $\mathbb{D}^{1},{ }^{1}$ Eungyeong Jang $\mathbb{D}^{2,3}$ and Jang-Hoon Lee $\mathbb{D}^{2}$ \\ ${ }^{1}$ Department of Clinical Korean Medicine Graduate School, Kyung Hee University, 26 Kyungheedae-ro, Dongdaemun-gu, \\ Seoul 02447, Republic of Korea \\ ${ }^{2}$ Department of Internal Medicine, College of Korean Medicine, Kyung Hee University, 26 Kyungheedae-ro, Dongdaemun-gu, \\ Seoul 02447, Republic of Korea \\ ${ }^{3}$ Department of Internal Medicine, Kyung Hee University Korean Medicine Hospital, 23 Kyungheedae-ro, Dongdaemun-gu, \\ Seoul 02447, Republic of Korea
}

Correspondence should be addressed to Jang-Hoon Lee; komclive@khmc.or.kr

Received 11 February 2020; Revised 29 June 2020; Accepted 6 July 2020; Published 29 July 2020

Academic Editor: Sokcheon Pak

Copyright $(2) 2020$ Soyeon An et al. This is an open access article distributed under the Creative Commons Attribution License, which permits unrestricted use, distribution, and reproduction in any medium, provided the original work is properly cited.

\begin{abstract}
Hepatobiliary disease currently serves as an important public health issue due to the fact that it is one of the major causes of death among economically active individuals and can easily progress to chronic diseases. Despite the development of vaccines and numerous drugs, a definite treatment remains lacking owing to different stages of the disease itself, its intricate pathogenesis, an effect uncertainty for long-term use, resistance, and side effects. Curcuma longa (C. longa), which belongs to the family Zingiberaceae and the genus Curcuma, has long been used not only as spice for curry or dye but also as a constituent of herbal formula for the treatment of different diseases due to its bioactive activities. Recently, many studies on the experimental results of $C$. longa have been published relative to hepatobiliary diseases such as fatty liver, hepatitis, cirrhosis, and tumors. Therefore, in this review, we aimed to summarize the pharmacological effects and underlying molecular mechanisms of C. longa and its four compounds, $\beta$-elemene, germacrone, ar-turmerone, and bisacurone, against hepatobiliary diseases. C. longa exhibited antioxidant, hepatoprotective, antisteatotic, anti-inflammatory, antifibrotic, antitumor, and cholagogic effects by regulating apoptosis, CYP2E1, Nrf, lipid metabolism-related factors, TGF- $\beta$, NF- $\kappa$ B, CYP7A1, and so on. In particular, $\beta$-elemene could be an attractive compound owing to its remarkable hepatoprotective, anti-inflammatory, antifibrotic, and antitumor activities. Altogether, the present review provides a preclinical basis for the efficacy of $C$. longa as an effective therapeutic agent for the prevention and treatment of hepatobiliary diseases, despite the need for further studies to establish the extraction conditions and separation of active constituents with high bioavailability, and warrants further evaluation in clinical trials.
\end{abstract}

\section{Introduction}

Hepatobiliary disease includes a wide spectrum of hepatic and biliary disorders which are caused by damages induced by toxic chemicals, drugs, alcohol consumption, viral infection, carcinogen, and obesity. Recently, acute and chronic diseases of the liver and biliary system have become a major public issue as current hepatobiliary diseases such as nonalcoholic fatty liver disease, drug-induced liver injury, hepatocellular carcinoma, and gallbladder, and biliary tract cancers are closely related to recent lifestyle changes [1]. Both males and females are vulnerable to different hepatobiliary diseases despite the sex disparity that exists in the prevalence of autoimmune diseases, gallstones, and hepatocarcinoma (HCC) and alcohol metabolism $[2,3]$. Unfortunately, most hepatobiliary diseases are diagnosed at the advanced stage where they have already progressed as most patients experience few signs or symptoms. Hence, the prevalence, mortality, and the health care burden of hepatobiliary diseases are steadily increasing. Approximately 2 million liver-related deaths occur every year worldwide [4]. However, the true global burden could be higher than expected. Although there has been evident breakthrough with curative agents over the last decades to manage hepatobiliary 
diseases, an optimal preventive and therapeutic strategy has yet to be established because of the several stages of the disease itself, complicated pathogenesis, low efficacy at longterm use, drug resistance, and undesirable side effects.

Preventing and treating hepatobiliary diseases with medicinal plants has had a long history. To date, many herbal medicines including Silybum marianum (milk thistle), Glycyrrhiza glabra, Phyllanthus amarus, Artemisia capillaris, Lysimachia christinae, and Lonicera japonica have been demonstrated to exhibit beneficial effects in managing different indications related to liver and biliary dysfunctions without harmful side effects [5, 6]. From the hepatoprotective and therapeutic viewpoints, Curcuma longa (C. longa) deserves clinical attention. According to the TCM theory, the radix (Yujin in Chinese) and rhizoma (Jiang Huang in Chinese) of C. longa are commonly attributed to the liver meridian [7]. Accumulating data have emphasized the pharmacological effects of $C$. longa for resolving pathological disorders in the hepatic, gallbladder, and biliary system. In addition, curcumin, a representative compound from C. longa, was introduced as a promising candidate that exhibits anti-inflammatory, antifibrotic, and antitumor activities which contribute to the treatment of viral hepatitis, stone, fibrosis, nonalcoholic steatohepatitis (NASH), primary sclerosing cholangitis (PSC), HCC, cholangiocarcinoma, etc. [8]. Due to the low absorption and bioavailability of curcumin, assuming that the effects of C. longa are largely attributed to the efficacy of curcumin might be misleading.

To date, a review article summarizing the preclinical evidence of $C$. longa or its active constituents, besides curcumin, against hepatobiliary diseases has not been published. Herein, we aimed to demonstrate available experimental results and the molecular mechanism of C. longa and its four constituents that are related to the prevention and treatment of hepatobiliary diseases.

\section{Pharmacological Activities of $C$. longa}

Liver disease represents a group of disorders characterized by stages of progression from steatosis and hepatitis to cirrhosis and cancer. This diverse spectrum of hepatic diseases is markedly associated with the pathophysiology of the biliary system [9]. According to the pathological stages of hepatobiliary disease, the pharmacological activities of $C$. longa can be generally classified into hepatoprotective, antioxidant, antisteatotic and antilipidemic, anti-inflammatory, antifibrotic, antitumor, and cholagogic effects.

2.1. Hepatoprotective Effect. The liver is a major organ in the management of metabolism, detoxification, and immune function. Moreover, it has a remarkable role of regenerating injured hepatocytes and restoring these cells to their original state. However, long-term exposure to different factors, such as virus, drugs, alcohol, and obesity, causes the liver to lose its restoration ability, eventually resulting in hepatitis, liver cirrhosis, and cancer. Although strong radical scavengers are widely applied to treat different liver diseases, the clinical efficacy of antioxidants is yet to reach a consensus [10]. Thus, the therapeutic agents that manage a variety of hepatic diseases need to exhibit hepatoprotective and antioxidant effects [11].

Carbon tetrachloride $\left(\mathrm{CCl}_{4}\right)$ is a representative toxic material that induces severe liver injury, which may result in hepatic cirrhotic changes. In rodent models, oral administration and intraperitoneal injection of $\mathrm{CCl}_{4}$ increased the levels of the biochemical indicators (alanine aminotransferase (ALT), aspartate aminotransferase (AST), lactate dehydrogenase (LDH), alkaline phosphatase (ALP), and total bilirubin (TB)) and the hepatosomatic index with hepatic lobular disorganization. C. longa reversed these abnormal measures and improved the histological changes [12-24]. The rhizomes of C. longa lowered the levels of serum AST, ALT, and ALP, hepatosomatic index, and mortality in rats with diethylnitrosamine- (DEN-) induced carcinogenic liver injury [25-27]. Furthermore, in the presence of thioacetamide (TAA) and aflatoxin, which are toxic carcinogens, C. longa recovered serum AST, ALT, protein, and albumin levels and decreased the hepatosomatic index in Sprague Dawley (SD) rats and broiler chickens, respectively [28, 29].

In addition to the above toxic substances, $C$. longa exerted hepatoprotective effects against alcohol [30-34], drugs [35-38], and NASH-induced methionine-choline deficient (MCD) diet [39]. The methanol extract of the rhizomes of $C$. longa exhibited a stronger cell viability in normal primary hepatocytes than curcuminoids [30], which suggests the potential of the noncurcuminoid ingredients to relieve alcohol toxicity. In drug-induced liver injury (DILI) caused by anti-inflammatory analgesic [35], antituberculosis [36], anticancer [37], and immunosuppressant [38] drugs, $C$. longa normalized the levels of AST, ALT, ALP, and TB, which are key indicators of the DILI assessment tool of the Council for International Organizations of Medical Sciences (CIOMS). C. longa improved histological liver injury and the serological measures against acetaminophen, a well-known DILI-inducing drug, in male SD rats [35].

C. longa exerted hepatoprotective properties against liver injury induced by heavy metals, such as lead [40] and mercury [41], and toxic pesticides, such as carbofuran [42] and endosulfan [43], by lowering the serum levels of AST, ALT, ALP, gamma-glutamyl transpeptidase (GGT), and $\mathrm{TB}$, improving hepatic protein synthesis, and preventing toxicity-induced weight loss in rats and chickens. Specifically, oral supplementation of C. longa $(500 \mathrm{mg} / \mathrm{kg}$ daily for 28 days) led to a significant reduction of the elevated AST, ALT, and ALP in Wistar albino rats, which are abnormal markers associated with the hepatocellular damage. Treatment with $C$. longa attenuated a significant increase of lipid peroxidation (LPO) and elevated the level of glutathione (GSH), which suggest that the possible molecular mechanism of pharmacological effects of C. longa against lead-induced hepatotoxicity might be involved in reducing oxidative stress [40]. In addition, the ethanol extract of the rhizomes of C. longa upregulated the expression of hepatic microsomal proteins that play a critical role in detoxification, which may contribute 
to its beneficial activity against $\mathrm{HgCl}_{2}$-induced hepatotoxicity in SD rats [41].

Therefore, C. longa protected the liver from different factors, such as chemicals, drugs, alcohol, heavy metals, and pesticides, which may increase the risk of liver injury, by inhibiting apoptosis and the normalization of serological and histological changes. Further studies focusing on the medicinal parts, extraction, and the chemical constituents of C. longa related to its hepatoprotective activities are required to strengthen its uses in the clinical settings.

2.2. Antioxidant Effect. Although blood tests and image inspections of patients with liver diseases reveal normal ranges, oxidative damage is frequently observed in the liver. For example, serum AST or ALT levels were normal or slightly elevated in obese fatty liver patients. However, there was a definite change in the oxidative stress markers of hepatic tissue [44]. Hence, the antioxidant effects of $C$. longa can play a crucial role in the management of hepatobiliary diseases because oxidative stress is closely associated with hepatic steatosis, inflammation process, cirrhosis, and tumorigenesis.

$\mathrm{CCl}_{4}[12-14,16-19,23]$, DEN [25], TAA [28], p-dimethylaminobenzaldehyde (p-DAB) [45], and benzopyrene [46] are the main reagents used to induce intrahepatic oxidative stress in most experiments performed to investigate the antioxidant effects of C. longa. Most of these chemicals are metabolized in the hepatic endoplasmic reticulum, and their metabolites lead to protein and lipid peroxidation, depletion of antioxidant enzymes, and triggering of hepatic necrosis by covalently binding with nuclear DNA [47]. First, C. longa increased the production of the antioxidant factors, such as superoxide dismutase (SOD), catalase (CAT), glutathione peroxidase (GSH-Px), and glutathione reductase (GR) in the liver of rodents, which were depleted by the above chemicals. In addition, $C$. longa regulated the intrahepatic malondialdehyde (MDA) levels, one of the most popular biomarkers of oxidative stress in rats administered TAA. Furthermore, the $95 \%$ ethanol extract of the rhizomes of C. longa reversed the levels of hepatic nitrotyrosine (a biological marker for protein oxidation) and urinary 8-hydroxy-2-deoxyguanosine (8-OH-dG), indicating DNA oxidative damage, as effective as silymarin in SD rats [28].

C. longa optimized the level of intrahepatic antioxidant molecules and reduced the production of lipid peroxidation against oxidative damage caused by alcohol $[19,33,34,39]$, drugs [17, 35, 37, 38, 48], MCD diet [39], pesticides [42, 43], heavy metals [40, 41], and iron [49]. The inhibition of intrahepatic cytochrome P450 2E1 (CYP2E1) by C. longa contributed to its antioxidant effects against alcohol-induced oxidative stress in C57BL/ 6 mice [33]. C. longa facilitated the reduction of $\mathrm{Fe}^{3+}$ ion in male New Zealand rabbits [49], which could contribute to the protection of liver tissue from oxidative damage caused by excess iron, during iron overload-induced liver injury.
Altogether, C. longa might exhibit strong antioxidant activities against the precursors, causing oxidative stress in the liver, such as chemicals, carcinogens, alcohol, drugs, pesticides, heavy metals, and iron, and two molecular mechanisms can be involved in its action. First, C. longa could markedly prevent and inhibit the overproduction of free radicals and lipid peroxides in the hepatic and gall bladder tissue by mediating a significant amount of CYP2E1 expression. CYP2E1 often generates reactive oxygen species, such as the superoxide anion radical and hydrogen peroxide, and it is frequently activated in chronic liver diseases [50]. Hence, further studies focusing on the antioxidant activities of C. longa via the regulation of intrahepatic CYP2E1 must be implemented to enable the use of this herb to treat hepatobiliary diseases. Second, C. longa exhibited some strengths in increasing the amount of antioxidant materials, and one of the underlying mechanisms might be the transcriptional regulation of nuclear factor erythroid 2-related factor 2 (Nrf2) targets. The rhizomes of C. longa increased intrahepatic Nrf2 levels in a dose-dependent manner against $\mathrm{CCl}_{4}$-induced injury. Additionally, antioxidant enzymes, such as SOD and CAT, were upregulated. Its efficacy to remove free radicals and lower lipid peroxidation is comparable to that of butylated hydroxytoluene (BHT) [12] and vitamin C [48], respectively. Moreover, C. longa lowered the intrahepatic accumulation of lipid peroxides and activated the antioxidant defense system without causing toxicity in normal Wistar rats [51]. Hence, C. longa might be employed to manage different hepatobiliary diseases owing to its antioxidant activities.

2.3. Antisteatotic and Antilipidemic Effects. The liver is in charge of lipid homeostasis by controlling the uptake and breakdown of dietary fatty acids for energy production, synthesizing de novo lipogenesis, or excretion from the liver [52]. Simple hepatic steatosis is defined as the intrahepatic accumulation of at least $5 \%$ of triglyceride (TG) of liver weight due to fatty acid surplus. Intrahepatic fat infiltration is itself the cause of NASH and accelerates the oxidative damage of hepatocytes, which makes the liver vulnerable to inflammation or fibrosis progression. In addition, hepatic steatosis is regarded as an independent risk factor of metabolic syndromes, such as insulin resistance, dyslipidemia, and cardiovascular diseases, and it has been reported to be more closely associated with metabolic syndromes than obesity [53].

The antisteatotic effects of $C$. longa were investigated in rodent models induced by $\mathrm{CCl}_{4}[13,14,21,23]$, highfructose diet [54, 55], and high-fat diet [54, 56, 57]. C. longa decreased the levels of TG contents, total cholesterol, and low-density lipoprotein (LDL) in the liver tissue, and hepatic histological findings by Oil Red O staining were consistently improved. Regarding the antisteatotic effects of C. longa, its water extract at $250^{\circ} \mathrm{C}$ impeded the uptake of fatty acids into the liver by suppressing the mRNA expression of CD36 and fatty acid transport protein (FATP) in C57BL/6 mice. In addition, it inhibited intrahepatic lipid synthesis via the regulation of sterol regulatory element-binding protein-1c 
(SREBP-1c), fatty acid synthase (FAS), and acetyl CoA carboxylase-1 (ACC) mRNA levels. Furthermore, it promoted lipolysis partially through the regulation of AMP-activated protein kinase (AMPK), peroxisome proliferator-activated receptor- $\alpha$ (PPAR- $\alpha$ ), and carnitine palmitoyltransferase-1 (CPT-1) mRNA levels [56]. Particularly, the rhizomes of $C$. longa facilitated the secretion of lipids from the liver into blood via the increase in hepatic PDI [13] and betaine [54] expression in male SD rats.

It is important to modulate the lipid contents that circulate in the blood and the inhibition of intrahepatic lipid accumulation for the treatment of hepatic steatosis. C. longa increased the level of serum high-density lipoprotein (HDL) and lowered the level of serum LDL, TG, and total cholesterol against high-fat diet [21, 57-59], carcinogen [26, 29,41,45,46], ethanol [34], and pesticides $[42,43]$. In addition, $C$. longa decreased the regions of aortic fatty streak in rabbits with atherosclerosis [58]. The modulatory effects of $C$. longa against serum lipids might inversely aid in the reduction of lipid inflow into the liver tissue.

In conclusion, $C$. longa can be developed as an important agent to treat fatty liver diseases, which account for a large portion of hepatobiliary diseases. In particular, the water extract of C. longa was found to exhibit strong antisteatotic and hypolipidemic effects, which were involved in the pharmacological mechanisms related to lipid metabolism.

2.4. Anti-Inflammatory Effect. Inflammation has a close interrelationship with oxidative stress and steatosis in the pathogenesis of hepatobiliary diseases. The management of inflammation is crucial in the treatment of hepatobiliary diseases because hepatitis can be regarded as a stage prior to the development of cirrhosis or cancer [60,61].

The anti-inflammatory effects of $C$. longa were mainly elucidated by a significant reduction in hepatic and serum levels of tumor necrosis factor- $\alpha$ (TNF- $\alpha$ ), which was elevated by drugs [35], ethanol [32], MCD diet [39], and TAA [28]. C. longa markedly decreased the hepatic interleukin-6 (IL-6) value in C57BL/6 mice administered alcohol [32] and an MCD diet [39], which are models that demonstrate the important role of IL- 6 in the development of alcoholic or nonalcoholic fatty liver into cirrhosis or cancer [62]. C. longa improved the major hallmark of the inflammation-associated histological findings, such as periportal inflammatory cell infiltration $[19,36,42]$, hepatic vascular congestion [34, 41], F4/80-positive macrophages [39], and mononuclear cellular infiltration [34].

These pharmacological activities of C. longa were mainly based on the decrease in inflammatory cytokine production by the inhibition of lipid peroxidation through its antioxidant actions. In a liver injury model induced by valproic acid and paracetamol, the water extract of $C$. longa lowered the intrahepatic MDA levels by increasing antioxidant enzymes, resulting in the suppression of TNF- $\alpha$ activity in the liver of male SD rats [35]. Similarly, C. longa augmented SOD and CAT levels and reduced the levels of intrahepatic MDA and serum TNF- $\alpha$ in a TAA-induced liver cirrhosis model [28].

Therefore, C. longa might exhibit strong anti-inflammatory effects against inflammatory reactions in the liver. However, its efficacy should be investigated in experimental models that mimic cholangitis or cholecystitis because curcumin alleviated sclerosing cholangitis in mice [63].

2.5. Antifibrotic Effect. Fibrosis involves excess extracellular matrix (ECM), such as collagen and fibronectin, and this production is initiated by the activation of hepatocellular stellate cells into myofibroblasts in the process of repairing damaged hepatocytes. Fibrosis can result in reduced liver function, hepatocyte necrosis, decreased intrahepatic microcirculation, and even cirrhosis or cancer [64]. However, because fibrosis can be reversed unlike liver cirrhosis, active management of liver fibrosis is required.

First, C. longa was found to reduce the amount of collagen, which was accumulated in the liver tissue. The hot water extract of the rhizomes of $C$. longa inhibited the excessive accumulation of alpha-1 type I collagen and the sirus-red positive area in the liver of mice fed an MCD diet [39]. Second, C. longa enhanced the expression of enzymes promoting collagen degradation in the liver tissue by inhibiting tissue inhibitor of metalloprotease (TIMP)-1, which blocks the activity of matrix metalloprotease-2 (MMP-2) [39]. Third, C. longa could markedly suppress hepatic alpha-smooth muscle actin ( $\alpha$-SMA), a specific protein marker of hepatic stellate cells (HSCs) contributing to the progression of liver cirrhosis [39]. The antifibrotic activities of $C$. longa may be exhibited via two molecular mechanisms - the blockade of transforming growth factor- $\beta$ (TGF- $\beta$ ) signaling pathway $[28,39]$ and the induction of apoptosis of HSCs [65].

Hence, C. longa reduced collagen accumulation, accelerated the decomposition of the ECM, and inhibited the activity of proteins involved in the progression of liver cirrhosis. In addition, the rhizomes of $C$. longa shortened the prolonged prothrombin time in TAA-induced SD rats [28]. The effects of $C$. longa against liver cirrhosis and its complications, such as delayed coagulation, should be investigated in further research. Regarding TGF- $\beta$ signaling suppression and proapoptosis of $C$. longa against hepatic fibrogenesis, PPAR- $\gamma$ activation can hinder binding to TGF$\beta$ and induce apoptosis of HSCs. Such findings suggest that C. longa could act as a PPAR- $\gamma$ agonist in the treatment of hepatobiliary diseases.

2.6. Antitumor Effect. Most experimental studies demonstrating the antitumor effects of $C$. longa against hepatobiliary system were based on HCC cell models or animal models induced by carcinogens of HCC. C. longa inhibited the proliferation of HepG2 cells by inducing apoptotic changes [66]. In addition, the rhizomes of $C$. longa lowered the HCC incidence rate [27] and the level of the serum tumor marker, $\alpha$-fetoprotein [26], in DENstimulated SD rats. In parallel, histological findings were characterized by the reduction in the number and size of 
GGT-positive hepatocytes, which are important in tumorigenesis in SD rats induced by DEN [26, 27]. Furthermore, C. longa impeded tumor angiogenesis by decreasing the level of serum vascular endothelial growth factor (VEGF) in DEN-induced Wistar rats [25]. In p-DAN and phenobarbital-induced rat models, the ethanol extract of the rhizomes of C. longa inhibited liver carcinogenesis by suppressing the hepatic expression of p53 and Bcl-2 and prevented cancer metastasis by disturbing hepatic MMP activity [45].

With regard to cholangiocarcinoma, C. longa displayed antitumor effects by upregulating the apoptosis of RMCCA1 cells via the activation of the mitogen-activated protein kinase (MAPK) signaling pathway [67].

In summary, C. longa might exert its antitumor effects on tumor markers, angiogenesis, and tumorigenesis through cell cycle arrest, Bcl-2 suppression, and MAPK signaling activation. Its anticancer activities can be applied to treat both HCC and bile duct cancer. Additionally, unlike conventional anticancer drugs, C. longa may have fewer side effects, such as weight loss and anorexia. Through further studies, C. longa might be demonstrated as a potent anticancer herb for the treatment of hepatobiliary cancer.

2.7. Cholagogic Effect. Cholestasis, a condition involving a decrease in bile flow, is caused by multiple factors, including infection, alcohol, drugs, tumor, and autoimmunity. Cholestasis can cause damage to organelles and cell membranes in hepatocytes and dysfunction in the hepatobiliary system, eventually resulting in symptom manifestation, such as jaundice, xanthoma, and itch [68]. Although antibiotics/ antivirals and immunosuppressants are used to treat infectious cholestasis and autoimmune cholestasis, respectively, ursodeoxycholic acid (UDCA) is currently recommended as the primary therapeutic drug to improve clinical symptoms and bile flow [69]. However, the development of drugs to treat cholestasis and prevent its complications is urgently required as the effects of UDCA remain limited.

C. longa elevated the total amounts of bile acids and bile secretion by activating the bile excretion pump in a concentration-dependent manner $[41,70,71]$. In particular, the water extract of the radix of $C$. longa increased the level of serum total bile acids by UDP-glucuronyl transferase activity for cholesterol excretion from the liver in high-fat diet rat models. This result is accompanied by the increased TG discharge to stool and decreased serum lipid contents [72]. Similarly, the water extract of the rhizomes of $C$. longa activated the cholesterol $7 \alpha$-hydroxylase (CYP7A1) enzyme converting cholesterol into bile acids in SD rats fed a high-fat diet [57].

In summary, C. longa exhibited cholagogic effects by increasing the production and secretion of total bile acids in high-fat diet-induced rats. However, there are no animal models induced by bile duct ligation or related to liver cirrhosis for evaluating the efficacy of C. longa. Therefore, different models stimulated by drugs, alcohol, autoimmune inflammation, viral infections, etc., are required to assess the pharmacological effects of $C$. longa in the treatment of cholestasis-induced dysfunctions.

\section{Pharmacological Activities of Active Compounds Isolated from C. longa}

The main constituents that contribute to the bioactive effects of $C$. longa on hepatobiliary disease are largely grouped as curcuminoids and noncurcuminoids [73]. Curcuminoids, which are lipophilic polyphenols, account for approximately $5 \%$ of C. longa. However, their efficacy has been constantly reported. In particular, curcumin, the principal curcuminoid, has demonstrated antioxidant, anti-inflammatory, antiviral, antifibrotic, and anticancer effects against hepatobiliary diseases [8]. However, extensive studies focusing on noncurcuminoids, such as elemene, germacrone, turmerone, and bisacurone, have recently accumulated [74]. Therefore, this review presents the pharmacological actions of the 4 noncurcuminoid constituents from $C$. longa which might have therapeutic effects against hepatobiliary diseases (Figure 1).

3.1. $\beta$-Elemene. $\beta$-Elemene (Figure $1(\mathrm{a}), \mathrm{C}_{15} \mathrm{H}_{24}$, $204.34 \mathrm{~g} / \mathrm{mol}$ ) is a member of the elemene sesquiterpenoids derived from the essential oil of $C$. longa [73]. Its compound exerted beneficial activities against various types of tumor, such as lung cancer, breast cancer, prostate cancer, cervical cancer, gastric cancer, and sarcoma cancer [75]. Similarly, most experimental studies of $\beta$-elemene targeting hepatobiliary diseases have been related to cancer. In mice models transplanted with human HCC cell lines (H22 [76] and MHCC97H cells [77]), $\beta$-elemene reduced the weight and volume of the tumor tissue. In particular, $\beta$-elemene enhanced the sensitivity of the anticancer drugs by increasing the level of copper transporter 1, aiding in the uptake of oxaliplatin into liver cells [77]. Based on the underlying mechanisms of its anticancer effects, $\beta$-elemene inhibited the proliferation of cancer cells by increasing histone $\mathrm{H} 1$ protein in $\mathrm{H} 22$ cells [76], and its treatment induced G2/M arrest and apoptosis of HepG2 cells by augmenting Fas and FasL mRNA and proteins on the cellular surface [75].

In addition to anticancer effects, $\beta$-elemene exerted anti-inflammatory effects by downregulating hepatic CD14 expression and suppressing serum TNF- $\alpha$ and endotoxin in CCl4-induced rats [78]. $\beta$-Elemene lowered the serum AST and ALT levels in rats with CCl4-induced liver damage [79]. In addition, $\beta$-elemene decreased dendrites and increased the vesicular structure of LX-2 cells, thereby reducing liver fibrosis [80]. Similarly, $\beta$-elemene injection was found to lower hepatic collagen deposition in $\mathrm{CCl}_{4}$-induced rats, which might be caused by the reduction in serum angiotensin-II level and hepatic angiotensin-II type 1 receptor mRNA level [79].

In summary, $\beta$-elemene might be one of the key active constituents of $C$. longa for the treatment of hepatobiliary 


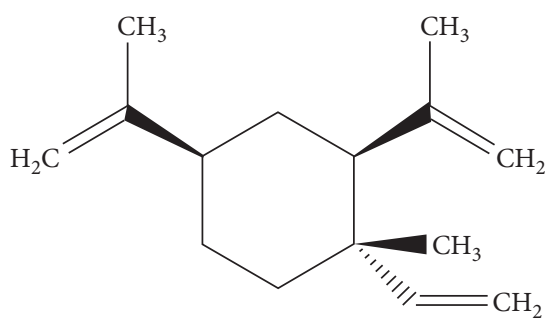

(a)

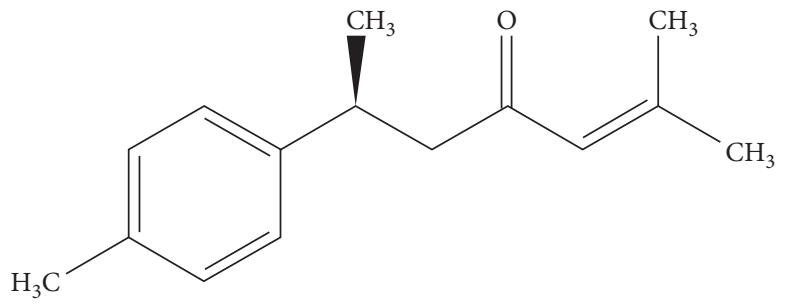

(c)

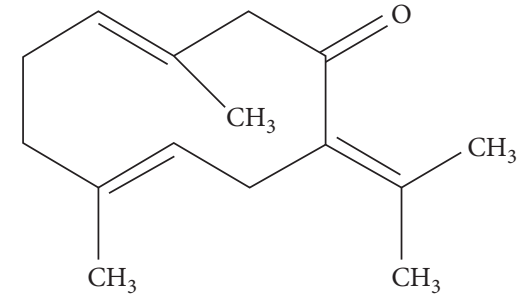

(b)

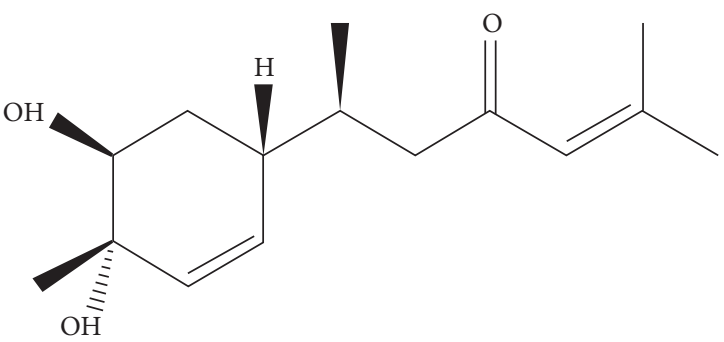

(d)

FIGURE 1: Four noncurcuminoid compounds obtained from C. longa. (a) $\beta$-Elemene $\left(\mathrm{C}_{15} \mathrm{H}_{24}\right.$, molecular weight (MW) of 204.34 g/mol). (b) Germacrone $\left(\mathrm{C}_{15} \mathrm{H}_{22} \mathrm{O}\right.$, MW of $\left.218.34 \mathrm{~g} / \mathrm{mol}\right)$. (c) Aromatic turmerone (ar-turmerone, $\mathrm{C}_{15} \mathrm{H}_{20} \mathrm{O}$, MW of $216.32 \mathrm{~g} / \mathrm{mol}$ ). (d) Bisacurone $\left(\mathrm{C}_{15} \mathrm{H}_{24} \mathrm{O}_{3}, \mathrm{MW}\right.$ of $\left.252.354 \mathrm{~g} / \mathrm{mol}\right)$.

diseases because it exerted inhibitory effects against liver injury, inflammation, and fibrosis in different experimental models.

3.2. Germacrone. Germacrone (Figure 1(b), $\mathrm{C}_{15} \mathrm{H}_{22} \mathrm{O}$, $218.34 \mathrm{~g} / \mathrm{mol}$ ) belongs to the volatile sesquiterpene family from C. longa. The pharmacological activities of germacrone in hepatobiliary diseases can be classified into two categories, namely, hepatoprotective and anticancer effects. Two in vivo experiments in Japan demonstrated that the intake of germacrone reversed the increase in the serum level of AST and ALT induced by D-galactosamine (D-GalN) in mice $[81,82]$. In human hepatoma cell lines, such as HepG2 and Bel7402, germacrone inhibited cellular proliferation by upregulating the apoptosis index. In particular, germacrone exhibited the induction of apoptosis similar to the control group in the normal liver cell line, LO2 cells, and significantly induced the apoptosis of HepG2 cells. These findings suggest that germacrone displays less toxic hepatoprotective effects and has strengths as it is only toxic to tumor cells [83].

Therefore, similar to $\beta$-elemene, germacrone is expected to not only possess hepatoprotective effects but also anticancer effects. Future research on biliary tract cancer and liver cancer must be conducted to identify its high potency against cancer with minor impacts on normal cells.

3.3. Ar-Turmerone. Aromatic turmerone (ar-turmerone, Figure $1(\mathrm{c}), \mathrm{C}_{15} \mathrm{H}_{20} \mathrm{O}, 216.32 \mathrm{~g} / \mathrm{mol}$ ) was introduced as a representative active compound of $C$. longa with curcumin [84]. A study conducted in Egypt reported that C. longa comprised more ar-turmerone than curcumin [66].

Ar-turmerone exhibited hepatoprotective, anticancer, and cholagogic effects against hepatobiliary diseases. Ar- turmerone improved the ethanol-induced reduction in the cell viability of hepatocytes isolated from SD male rats [30]. In normal Wistar rats, intraduodenal injection of ar-turmerone increased the volume of total bile acids and bile secretion [71]. In addition, ar-turmerone caused significant inhibitory effects on the cell proliferation of three HCC cells, namely, HepG2, Huh-7, and Hep3B. In particular, ar-turmerone induced both regulation of Bax and p53 upregulated modulator of apoptosis (PUMA) of the intrinsic apoptotic pathway and caspase activation of the extrinsic apoptotic pathway [85].

In recent studies, ar-turmerone enhanced the immune system by elevating the number of monocytes in peripheral blood [86] and exhibited antiangiogenic effects on HMEC-1 cells, zebrafish, and matrigel plug mice [87]. Hence, further in-depth and extensive studies need to be performed to demonstrate its potential as an antitumor drug against hepatobiliary cancer.

3.4. Bisacurone. Bisacurone (Figure $1(\mathrm{~d}), \mathrm{C}_{15} \mathrm{H}_{24} \mathrm{O}_{3}$, $252.354 \mathrm{~g} / \mathrm{mol}$ ) is more separated from the rhizomes of $C$. longa than its radix [88]. Recent studies have demonstrated the superior pharmacological effects of bisacurone compared to curcuminoids and other active ingredients in C. longa. For example, curcumin did not cause significant cell viability at a low concentration of $1 \mu \mathrm{M}$, while bisacurone at the same concentration caused high cell viability following ethanol treatment in hepatocytes isolated from SD rats [30]. Additionally, bisacurone was more effective at promoting bile secretion and produced more total bile acids than curcuminoids and ar-turmerone in Wistar rats [71]. A significant decrease in the ALT level in rat serum, which was increased by ethanol administration, was observed after a single intake of bisacurone [32]. 


\begin{tabular}{|c|c|c|c|c|c|c|}
\hline \multicolumn{7}{|c|}{ Curcuma longa } \\
\hline Hepatoprotective & Antioxidant & Antisteatotic/antilipidemic & Anti-inflammatory & Antifibrotic & Antitumor & Cholagogic \\
\hline $\begin{array}{l}\mathrm{CCL}_{4}, \mathrm{DEN}, \mathrm{TAA}, \\
\mathrm{MCD} \text {, alcohol, } \\
\text { drugs, lead, } \mathrm{HgCl}_{2}, \\
\text { carbofuran, } \\
\text { endosulfan }\end{array}$ & $\begin{array}{c}\mathrm{CCL}_{4}, \mathrm{DEN}, \mathrm{TAA}, \\
\text { aflatoxin, alcohol, drugs, } \\
\mathrm{MCD} \text {, high fat, lead, } \\
\mathrm{HgCl}_{2}, \mathrm{Fe}^{2+} \text {, benzopyrene, } \\
\text { carbofuran, endosulfan }\end{array}$ & $\begin{array}{l}\mathrm{CCl}_{4}, \mathrm{DEN}, \text { high } \\
\text { fructose and fat, } \\
\text { alcohol, drugs, } \\
\text { aflatoxin, benzopyrene, } \\
\text { carbofuran }\end{array}$ & $\begin{array}{l}\mathrm{CCl}_{4}, \mathrm{TAA}, \mathrm{MCD} \\
\text { aflatoxin, alcohol, } \\
\text { drugs, } \mathrm{HgCl}_{2} \\
\text { carbofuran }\end{array}$ & TAA, MDA & DEN, drugs & $\mathrm{HgCl}_{2}$, high fat \\
\hline $\begin{array}{l}\text { HepG2/CYP2E1, } \\
\text { primary hepatocytes, } \\
\text { Wister rats, SD rats, } \\
\text { Kunming mice, } \\
\text { albino mice, } \\
\text { ICR mice, DDI/Tohi } \\
\text { mice, C57BL/6J mice, } \\
\text { chickens, pigs }\end{array}$ & $\begin{array}{c}\text { HepG2, } \\
\text { HepG2/CYP2E1, } \\
\text { Wistar rats, SD rats, } \\
\text { albino mice, } \\
\text { Kunming mice, } \\
\text { ICR mice, } \\
\text { C57BL/6J mice, } \\
\text { rabbits, chickens }\end{array}$ & $\begin{array}{l}\text { Wistar rats, SD } \\
\text { rats, albino mice, } \\
\text { Kunming mice, } \\
\text { ICR mice, } \\
\text { Balb/c mice, } \\
\text { C57BL/6J mice, } \\
\text { rabbits, chickens }\end{array}$ & $\begin{array}{l}\text { Wistar rats, SD } \\
\text { rats, albino mice, } \\
\text { C57BL/6J mice, } \\
\text { ICR mice, pigs, } \\
\text { chickens }\end{array}$ & $\begin{array}{l}\mathrm{LX}-2 \text {, } \\
\text { SD rats, } \\
\text { C57BL/6J } \\
\text { mice }\end{array}$ & $\begin{array}{l}\text { HepG2, RMCCA1, } \\
\text { wistar rats, } \\
\text { SD rats, } \\
\text { white rats }\end{array}$ & $\begin{array}{l}\text { Wistar rats, } \\
\text { SD rats }\end{array}$ \\
\hline $\begin{array}{l}\downarrow \text { AST, ALT, LDH, } \\
\text { ALP, GGT, TB } \\
\uparrow \text { albumin } \\
\downarrow \text { hepatosomatic } \\
\text { index, mortality } \\
\text { and histological } \\
\text { improvement }\end{array}$ & $\begin{array}{c}\uparrow \text { SOD, CAT, GSH-Px, } \\
\text { GST } \\
\downarrow \text { ROS, NO, MDA, } \\
\text { 8-OH-dG, nitrotyrosine } \\
\uparrow \mathrm{Fe}^{3+} \text { reducing power }\end{array}$ & $\begin{array}{c}\downarrow \text { TG, TC, LDL } \\
\uparrow \text { HDL } \\
\downarrow \text { hepatic fat }\end{array}$ & $\begin{array}{l}\downarrow \text { TNF- } \alpha \text {, IL-6, IL-1 } \beta \\
\text { and histological } \\
\text { improvement }\end{array}$ & $\begin{array}{c}\downarrow \text { collagen I, TIMP- } 1, \\
\alpha \text {-SMA, prothrombin } \\
\text { time } \\
\text { and histological } \\
\text { improvement }\end{array}$ & $\begin{array}{l}\downarrow \text { VEGF, AFP, } \\
\text { incidence, MMPs, } \\
\text { tumor weight, } \\
\text { and histological } \\
\text { improvement }\end{array}$ & $\begin{array}{c}\uparrow \text { total bile acids } \\
\text { bile secretion/flow }\end{array}$ \\
\hline$\downarrow$ apoptosis & $\begin{array}{l}\downarrow \text { CYP2E1 } \\
\uparrow \text { Nrf2 }\end{array}$ & $\begin{array}{c}\downarrow \text { Cd } 36, \text { FATP, SREBP- } 1 \mathrm{c}, \\
\text { FAS, ACC, HMG-CoA } \\
\text { reductase } \\
\uparrow \text { AMPK, PPAR } \alpha, \text { CPT- } 1 \text {, } \\
\text { PDI, betaine }\end{array}$ & & $\begin{array}{c}\downarrow \text { TGF- } \beta \\
\uparrow \text { apoptosis }\end{array}$ & $\begin{array}{c}\downarrow \text { NF- } \kappa \text { B, MAPK } \\
\uparrow \text { apoptosis }\end{array}$ & $\begin{array}{c}\downarrow \text { CYP7A1 } \\
\uparrow \text { UDP-glucuronyl } \\
\text { transferase }\end{array}$ \\
\hline
\end{tabular}

Figure 2: Pharmacological activities of C. longa related to hepatobiliary diseases. C. longa exhibited hepatoprotective, antioxidant, antisteatotic/hypolipidemic, anti-inflammatory, antifibrotic, antitumor, and cholagogic effects via regulating apoptosis, CYP2E1, Nrf, lipid metabolism-related signaling factors, TGF- $\beta$, NF- $\kappa$ B, CYP7A1, and so on.

Accordingly, bisacurone might exhibit hepatoprotective effects on liver injury induced by ethanol intake and cholestasis. Furthermore, its efficacy could be stronger than that of curcuminoids and ar-turmerone.

\section{Discussion}

In this review, the experimental evidence of $C$. longa and its noncurcuminoid constituents $(\beta$-elemene, germacrone, arturmerone, and bisacurone), their pharmacological activities, and their underlying mechanisms that might contribute to the prevention and treatment of different hepatobiliary diseases, was summarized.

The causative factors resulting in hepatobiliary damage include alcohol, drugs, pesticides, heavy metals, excessive accumulation of fats, viral infections, exposure to toxic chemicals or carcinogens, and abnormalities in the immune system. These triggers might stimulate the emergence of the pathological phenomenon in the hepatobiliary system, such as structural and functional damages of tissues, steatosis, inflammation, fibrosis, and tumor [89]. The hepatobiliary system is vulnerable to oxidative stress. Excessive production of free radicals in the mitochondria, peroxisome, and microsomes of the liver parenchymal cells damages Kupffer cells, endothelial cells, and HSCs, as well as parenchymal cells themselves [10]. In addition, the reaction of bile components with free radicals affects the function of the gall bladder, which can cause some hepatobiliary diseases. Hence, resolving the oxidant/antioxidant imbalance and preventing toxic damage to cells and tissues are important to manage hepatobiliary diseases.
C. longa displayed its potency in regulating the oxidant/ antioxidant disparity and exhibiting hepatoprotective effects. These pharmacological effects might be activated through the regulation of CYP2E2 and Nrf expression, antiapoptosis, and detoxification of protein generation in microsomes of the liver. C. longa significantly altered steatosis, hyperlipidemia, and inflammatory reactions which occurred in the hepatobiliary system. Furthermore, it could markedly suppress fibrosis, tumorigenesis, and cholestasis by inhibiting TGF- $\beta$ signaling, the NF- $\kappa \mathrm{B}$ pathway, and CYP7A1 activity (Figure 2).

Based on these preclinical effects of C. longa, it can be used as therapeutic candidates for the treatment of toxic hepatitis, alcoholic liver disease, nonalcoholic fatty liver disease, cirrhosis, liver cancer, and cholestatic liver diseases. Regarding the molecular mechanism of C. longa in treating hepatobiliary diseases, further investigations on its role as a PPAR- $\gamma$ agonist are needed, which might contribute to regulating inflammation, fibrosis, and cancer. Although the optimal extraction method of C. longa is inconclusive, it is necessary to find an extraction process which can maximize the acquisition of its active constituents.

With regard to the four compounds belonging to the noncurcuminoid group obtained from C. longa, the hepatoprotective effects were commonly observed when all four constituents were administered. In particular, ar-turmerone and bisacurone protected hepatic parenchymal cells from alcohol-induced damage. Germacrone and $\beta$-elemene were found to exhibit hepatoprotective actions against $\mathrm{D}-\mathrm{GalN}$ and $\mathrm{CCl}_{4}$, respectively. In addition, $\beta$-elemene, germacrone, and ar-turmerone promoted the apoptosis of HepG2 cells, 


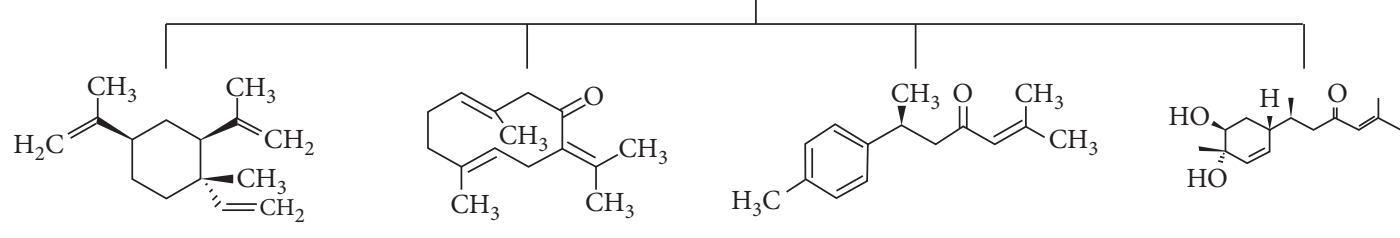

$\beta$-Elemene $\quad$ Germacrone

Ar-turmerone

\section{Hepatoprotective}

$\uparrow$ hepatocyte viability

(from ethanol)

Antitumor

Antitumor

$\uparrow$ HepG2 apoptosis

$\uparrow$ Bel7402 apoptosis

HepG2 apoptosis

( $\uparrow$ Fas and Fas L)

$\uparrow \mathrm{H} 22$ histone $\mathrm{H} 1$

$\uparrow$ MHCC97H copper transporter 1

\section{Hepatoprotective}

$\downarrow$ rat serum AST, ALT by $\mathrm{CCl}_{4}$

\section{Anti-inflammatory}

$\downarrow$ rat serum TNF- $\alpha$, endotoxin by $\mathrm{CCl}_{4}$

$\downarrow$ rat hepatic CD14 by $\mathrm{CCl}_{4}$

\section{Antifibrotic}

$\downarrow$ rat serum ANG II by $\mathrm{CCl}_{4}$

$\downarrow$ rat hepatic collagen and $\mathrm{AT}_{1}$ receptor

\section{Antitumor}

$\downarrow$ mice hepatoma size and weight

$\uparrow$ HepG2 apoptosis

$\uparrow$ Huh7 proliferation

$\uparrow$ Hep3B proliferation

\section{Hepatoprotective}

$\downarrow$ mice serum AST, ALT by D-GalN

\section{Cholagogic}

$\uparrow$ rat bile secretion

$\uparrow$ total bile acids
Bisacurone

\section{Hepatoprotective}

$\uparrow$ hepatocyte viability

(from ethanol)
Hepatoprotective

$\downarrow$ mice serum ALT by ethanol

Cholagogic

$\uparrow$ rat bile secretion

$\uparrow$ total bile acids

Figure 3: Pharmacological activities of four chemical compounds from C. longa. Four sorts of noncurcuminoid ingredients obtained from C. longa showed hepatoprotective effects commonly. Among them, $\beta$-elemene could be an attractive compound to treat different hepatobiliary diseases because it was excellent in anti-inflammatory, antifibrotic, and antitumor activities, as well as hepatoprotective effects.

and ar-turmerone inhibited the proliferation of Huh7 and Hep3B cells. The combination of $\beta$-elemene and conventional anticancer drugs has been suggested because it was found to suppress $\mathrm{H} 22$ cell proliferation by activating the histone $\mathrm{H} 1$ protein and elevating the sensitivity of anticancer drugs in MHCC $97 \mathrm{H}$ cells. $\beta$-Elemene has been approved as a Class II noncytotoxic therapeutic antitumor agent by the China Food and Drug Administration because of its low toxicity, high efficacy, and immune-enhancing effects [90] (Figure 3).

Interestingly, the C. longa extracts exhibited various pharmacological effects when administered to treat hepatobiliary diseases, despite the low bioavailability of curcumin, a representative ingredient of $C$. longa. Such finding infers that $C$. longa may contain active compounds, besides curcumin, that contribute to its efficacy. $\beta$-Elemene might serve as a strong candidate given its hepatoprotective, antiinflammatory, antifibrotic, and anticancer effects.

\section{Conclusions}

To our knowledge, this is the first review to summarize the therapeutic activities and pharmacological mechanisms of $C$. longa and its four active constituents, namely, $\beta$-elemene, germacrone, ar-turmerone, and bisacurone, in experimental models that mimic hepatobiliary diseases. This review presents available evidence regarding C. longa and may facilitate its use in the clinic to treat hepatobiliary diseases. However, several limitations exist regarding its use. First, its chemical compounds differ according to the cultivation area, harvest time, and the extraction method. Second, because its four ingredients are essential oils obtained from steam distillation, they have low solubility and poor absorption after ingestion like curcuminoids [74]. Therefore, based on this review, more efforts can be dedicated to broadening the range of clinical application of C. longa for treating different hepatobiliary diseases, such as the separation of its active ingredients with high bioavailability and efficacy, its standardization, and the establishment of optimal extraction conditions to maximize its effects.

\section{Conflicts of Interest}

The authors declare that there are no conflicts of interest regarding the publication of this paper.

\section{Authors' Contributions}

Soyeon An and Eungyeong Jang contributed equally to this work. 


\section{References}

[1] L. A. Torre, R. L. Siegel, F. Islami, F. Bray, and A. Jemal, "Worldwide burden of and trends in mortality from gallbladder and other biliary tract cancers," Clinical Gastroenterology and Hepatology, vol. 16, no. 3, pp. 427-437, 2018.

[2] M. L. Borum, "Hepatobiliary diseases in women," Medical Clinics of North America, vol. 82, no. 1, pp. 51-75, 1998.

[3] V. W. Keng, D. A. Largaespada, and A. Villanueva, "Why men are at higher risk for hepatocellular carcinoma?" Journal of Hepatology, vol. 57, no. 2, pp. 453-454, 2012.

[4] A. M. Moon, A. G. Singal, and E. B. Tapper, "Contemporary epidemiology of chronic liver disease and cirrhosis," Clinical Gastroenterology and Hepatology, 2019, In press.

[5] F. Stickel and D. Schuppan, "Herbal medicine in the treatment of liver diseases," Digestive and Liver Disease, vol. 39, no. 4, pp. 293-304, 2007.

[6] T. Gan, J. Chen, S. J. Jin, and Y. Wang, "Chinese medicinal herbs for cholelithiasis," Cochrane Database of Systematic Reviews, vol. 6, 2013.

[7] Y. Zhou, M. Xie, Y. Song et al., "Two traditional Chinese medicines curcumae radix and curcumae rhizoma: an ethnopharmacology, phytochemistry, and pharmacology review," Evidence-Based Complementary and Alternative Medicine, vol. 2016, Article ID 4973128, 30 pages, 2016.

[8] R. W. Hu, E. J. Carey, K. D. Lindor, and J. H. Tabibian, "Curcumin in hepatobiliary disease: pharmacotherapeutic properties and emerging potential clinical applications," Annals of Hepatology, vol. 16, no. 6, pp. 835-841, 2017.

[9] I. G. Rajapaksha, P. W. Angus, and C. B. Herath, "Current therapies and novel approaches for biliary diseases," World Journal of Gastrointestinal Pathophysiology, vol. 10, no. 1, pp. 1-10, 2019.

[10] S. Li, H.-Y. Tan, N. Wang et al., "The role of oxidative stress and antioxidants in liver diseases," International Journal of Molecular Sciences, vol. 16, no. 11, pp. 26087-26124, 2015.

[11] P. Saha, A. D. Talukdar, R. Nath et al., "Role of natural phenolics in hepatoprotection: a mechanistic review and analysis of regulatory network of associated genes," Frontiers in Pharmacology, vol. 10, pp. 1-25, 2019.

[12] R. Serairi Beji, R. Ben Mansour, I. Bettaieb Rebey et al., "Does Curcuma longa root powder have an effect against CCl4induced hepatotoxicity in rats: a protective and curative approach," Food Science and Biotechnology, vol. 28, no. 1, pp. 181-189, 2018.

[13] H. Y. Lee, S. W. Kim, G. H. Lee et al., "Curcumin and Curcuma longa L. extract ameliorate lipid accumulation through the regulation of the endoplasmic reticulum redox and ER stress," Scientific Reports, vol. 7, no. 1, pp. 1-14, 2017.

[14] Y. Yu, W. Xiong, S. Yu et al., "Protective effect of extract powder of turmeric on carbon tetrachloride induced acute hepatic injury in mice," Agricultural Science \& Technology, vol. 18, no. 11, pp. 2155-2162, 2017.

[15] J. Li, T. Lu, C. Mao, D. Ji, L. Li, and Y. Xiao, "Comparison on effect of Curcuma Rhizoma before and after processed with vinegar on hepatic fibrosis in rats induced by CCl4 composited factors," Chinese Traditional and Herbal Drugs, vol. 44, no. 19, pp. 2710-2716, 2013.

[16] G. H. Lee, H. Y. Lee, M. K. Choi, H. W. Chung, S. W. Kim, and H. J. Chae, "Protective effect of Curcuma longa L. Extract on $\mathrm{CCl} 4$-induced acute hepatic stress," BMC Research Notes, vol. 10, no. 1, pp. 1-9, 2017.

[17] H.-S. Lee, L. Li, H. K. Kim et al., "The protective effects of Curcuma longa Linn. Extract on carbon tetrachloride-induced hepatotoxicity in rats via upregulation of Nrf2," Journal of Microbiology and Biotechnology, vol. 20, no. 9, pp. 1331-1338, 2010.

[18] L. Subramanian and R. Selvam, "Prevention of CCI4 - induced hepatotoxicity by aqueous extract of turmeric," Nutrition Research, vol. 19, no. 3, pp. 429-441, 1999.

[19] Y. Kim, Y. You, H.-G. Yoon et al., "Hepatoprotective effects of fermented Curcuma longa L. on carbon tetrachloride-induced oxidative stress in rats," Food Chemistry, vol. 151, pp. 148-153, 2014.

[20] M. Sengupta, G. D. Sharma, and B. Chakraborty, "Hepatoprotective and immunomodulatory properties of aqueous extract of Curcuma longa in carbon tetra chloride intoxicated Swiss albino mice," Asian Pacific Journal of Tropical Biomedicine, vol. 1, no. 3, pp. 193-199, 2011.

[21] J.-K. Kang, H.-J. Kang, J.-H. Seo et al., "Effects of fermented turmeric (Curcuma longa) by Bacillus natto supplementation on liver function and serum lipid parameters in mice," Journal of the Korean Society of Food Science and Nutrition, vol. 38, no. 4, pp. 430-435, 2009.

[22] Y. Kiso, Y. Suzuki, C. Konno, H. Hikino, I. Hashimoto, and Y. Yagi, "Application of carbon tetrachloride-induced liver lesion in mice for screening of liver protective crude drugs," The Japanese Journal of Pharmacognosy, vol. 36, no. 3, pp. p238-p244, 1982.

[23] H. Y. Lee, J. B. Weon, Y. S. Jung, N. Y. Kim, M. K. Kim, and C. J. Ma, "Cognitive-enhancing effect ofAronia melanocarpaExtract against memory impairment induced by scopolamine in mice," Evidence-Based Complementary and Alternative Medicine, vol. 2016, no. 1, pp. 1-7, 2016.

[24] C. R. Kim, "Enhancement of liver function by Curcuma extract on acute hepatotoxicity in rat," Korean Journal for Food Science of Animal Resources, vol. 26, no. 3, pp. 386-393, 2006.

[25] M. El-Shahat, S. El-Abd, M. Alkafafy, and G. El-Khatib, "Enhancement of liver function by Curcuma extract on acute hepatotoxicity in rat (Commiphora molmol) vs. turmeric (Curcuma longa)," Acta Hstochemica, vol. 114, no. 5, pp. 421-428, 2006.

[26] T.-S. Jung and C.-W. Choi, "The effect of the curcumae longae rhizoma (CLR) extract on the hepatocellular carcinogenesis and acute liver damage induced by diethylnitrosamine (DENA) and CCl4in rats," Herbal Formula Science, vol. 22, no. 1, pp. 177-192, 2014.

[27] R. Thapliyal, K. N. Naresh, K. V. K. Rao, and G. B. Maru, "Inhibition of nitrosodiethylamine-induced hepatocarcinogenesis by dietary turmeric in rats," Toxicology Letters, vol. 139, no. 1, pp. 45-54, 2003.

[28] S. M. Salama, M. A. Abdulla, A. S. AlRashdi, A. H. A. Hadi, S. S. Alkiyumi, and S. Golbabapour, "Mechanism of hepatoprotective effect ofBoesenbergia rotundain thioacetamideinduced liver damage in rats," Evidence-Based Complementary and Alternative Medicine, vol. 2013, no. 1, pp. 1-13, 2013.

[29] M. Gholami-Ahangaran, N. Rangsaz, and S. Azizi, "Evaluation of turmeric (Curcuma longa) effect on biochemical and pathological parameters of liver and kidney in chicken aflatoxicosis," Pharmaceutical Biology, vol. 54, no. 5, pp. 780-787, 2015.

[30] C. Megumi, K. Muroyama, H. Sasako, and N. Tsuge, "Preventive activity of ar-turmerone and bisacurone isolated from turmeric extract against ethanol-induced hepatocyte injury," Food Science and Technology Research, vol. 23, no. 2, pp. 275-281, 2017. 
[31] H. Sung, Y.-H. Lee, and W. Jun, "In vitro hepatoprotective effects of fermented Curcuma longa L. by Aspergillus oryzae against alcohol-induced oxidative stress," Journal of the Korean Society of Food Science and Nutrition, vol. 45, no. 6, pp. 812-818, 2016.

[32] R. Uchio, Y. Higashi, Y. Kohama et al., "A hot water extract of turmeric (Curcuma longa) suppresses acute ethanol-induced liver injury in mice by inhibiting hepatic oxidative stress and inflammatory cytokine production," Journal of Nutritional Science, vol. 6, no. 3, pp. 1-9, 2017.

[33] M. Lee, Y. Kim, H.-G. Yoon et al., "Prevention of ethanolinduced hepatotoxicity by fermented Curcuma longa L. in C57BL/6 mice," Food Science and Biotechnology, vol. 23, no. 3, pp. 925-930, 2014.

[34] S. O. Nwozo, D. A. Osunmadewa, and B. E. Oyinloye, “Antifatty liver effects of oils from Zingiber officinale and Curcuma longa on ethanol-induced fatty liver in rats," Journal of Integrative Medicine, vol. 12, no. 1, pp. 59-65, 2014.

[35] H. S. Zaky, A. M. Gad, E. Nemr, W. Hassan, O. M. Abd ElRaouf, and A. A. Ali, "Modulatory effects of some natural products on hepatotoxicity induced by combination of sodium valproate and paracetamol in rats," Journal of Biochemical and Molecular Toxicology, vol. 32, no. 7, pp. 1-7, 2018.

[36] M. R. Adhvaryu, N. Reddy, and M. H. Parabia, "Effects of four Indian medicinal herbs on Isoniazid-, Rifampicin- and Pyrazinamide-induced hepatic injury and immunosuppression in Guinea pigs," World Journal of Gastroenterology, vol. 13, no. 23, pp. 3199-3205, 2007.

[37] R. H. Mohamad, A. M. El-Bastawesy, Z. K. Zekry et al., "The role of Curcuma longa against doxorubicin (adriamycin)induced toxicity in rats," Journal of Medicinal Food, vol. 12, no. 2, pp. 394-402, 2009.

[38] A. R. Moghadam, S. Tutunchi, A. Namvaran-Abbas-Abad et al., "Pre-administration of turmeric prevents methotrexateinduced liver toxicity and oxidative stress," BMC Complementary and Alternative Medicine, vol. 15, no. 1, pp. 1-13, 2015.

[39] R. Uchio, S. Murosaki, and H. Ichikawa, "Hot water extract of turmeric (Curcuma longa) prevents non-alcoholic steatohepatitis in mice by inhibiting hepatic oxidative stress and inflammation," Journal of Nutritional Science, vol. 7, no. 36, pp. 1-11, 2018.

[40] S. L. Baxla, R. Gora, R. H. Gora et al., "Hepatoprotective effect of Curcuma longa against lead induced toxicity in Wistar rats," Veterinary World, vol. 6, no. 9, pp. 664-667, 2013.

[41] D. Joshi, D. K. Mittal, S. Shukla, S. K. Srivastav, and V. A. Dixit, "Curcuma longa Linn. extract and curcumin protect CYP 2E1 enzymatic activity against mercuric chloride-induced hepatotoxicity and oxidative stress: a protective approach," Experimental and Toxicologic Pathology, vol. 69, no. 6, pp. 373-382, 2017.

[42] M. S. Hossen, E. M. Tanvir, M. B. Prince et al., "Protective mechanism of turmeric (Curcuma longa) on carbofuran-induced hematological and hepatic toxicities in a rat model," Pharmaceutical Biology, vol. 55, no. 1, pp. 1937-1945, 2017.

[43] M. M. Alagawany, M. R. Farag, and K. Dhama, "Nutritional and biological effects of turmeric (Curcuma longa) supplementation on performance, serum biochemical parameters and oxidative status of broiler chicks exposed to endosulfan in the diets," Asian Journal of Animal and Veterinary Advances, vol. 10, no. 2, pp. 86-96, 2015.

[44] L. A. Videla, R. Rodrigo, M. Orellana et al., "Oxidative stressrelated parameters in the liver of non-alcoholic fatty liver disease patients," Clinical Science, vol. 106, no. 3, pp. 261-268, 2004.

[45] A. R. Khuda-Bukhsh, A. Banerjee, R. Biswas, S. Pathak, N. Boujedaini, and P. Belon, "Crude extract of turmeric reduces hepato-toxicity and oxidative stress in rats chronically fed carcinogens," Journal of Complementary and Integrative Medicine, vol. 5, no. 1, pp. 1-38, 2008.

[46] S. Y. Cho, Y. G. Lee, and J. S. Lee, "The effect of curcumae rhizoma on benzo(a)pyrene induced hepatotoxicity," Journal of Korean Environmental and Hygienic Science, vol. 2, no. 1, pp. 145-150, 1992.

[47] A. M. Hruszkewycz, E. A. Glende Jr., and R. O. Recknagel, "Destruction of microsomal cytochrome P-450 and glucose6-phosphatase by lipids extracted from peroxidized microsomes," Toxicology and Applied Pharmacology, vol. 46, no. 3, pp. 695-702, 1987.

[48] M. R. Khazdair, R. Mohebbati, S. Karimi, A. Abbasnezhad, and M. Haghshenas, "The protective effects of Curcuma longa extract on oxidative stress markers in the liver induced by Adriamycin in rat," Physiology and Pharmacology, vol. 20, no. 1, pp. 31-37, 2016.

[49] A. C. Reddy and B. Lokesh, "Effect of dietary turmeric (Curcuma longa) on iron-induced lipid peroxidation in the rat liver," Food and Chemical Toxicology: An International Journal Published for the British Industrial Biological Research Association, vol. 32, no. 3, pp. 279-283, 1994.

[50] M. Marí, D. Wu, N. Nieto, and A. I. Cederbaum, "CYP2E1dependent toxicity and up-regulation of antioxidant genes," Journal of Biomedical Science, vol. 8, no. 1, pp. 52-58, 2001.

[51] A. Rezaei-Moghadam, D. Mohajeri, B. Rafiei et al., "Effect of turmeric and carrot seed extracts on serum liver biomarkers and hepatic lipid peroxidation, antioxidant enzymes and total antioxidant status in rats," BioImpacts, vol. 2, no. 3, p. 151, 2012.

[52] P. Nguyen, V. Leray, M. Diez et al., "Liver lipid metabolism," Journal of Animal Physiology and Animal Nutrition, vol. 92, no. 3, pp. 272-283, 2008.

[53] E. Fabbrini and F. Magkos, "Hepatic steatosis as a marker of metabolic dysfunction," Nutrients, vol. 7, no. 6, pp. 49955019, 2015.

[54] F. Tranchida, Z. Rakotoniaina, L. Shintu et al., "Hepatic metabolic effects of Curcuma longa extract supplement in high-fructose and saturated fat fed rats," Scientific Reports, vol. 7, no. 1, pp. 1-13, 2017.

[55] A. Hayashi, "Effect on lipid metabolism by turmeric (Curcuma longa Linn) supplementation in high fructose-diet-fed rats," Bulletin of Tokyo Family University, vol. 54, no. 2, pp. 13-18, 2014.

[56] J. Mun, S. Kim, H. G. Yoon et al., "Water extract of Curcuma longa L. Ameliorates non-alcoholic fatty liver disease," $\mathrm{Nu}$ trients, vol. 11, no. 10, pp. 1-13, 2019.

[57] W. F. Yiu, P. L. Kwan, C. Y. Wong et al., "Attenuation of fatty liver and prevention of hypercholesterolemia by extract of Curcuma longa through regulating the expression of CYP7A1, LDL-receptor, HO-1, and HMG-CoA reductase," Journal of Food Science, vol. 76, no. 3, pp. 80-89, 2011.

[58] J. L. Quiles, M. D. Mesa, C. L. Ramírez-Tortosa et al., "Curcuma longa extract supplementation reduces oxidative stress and attenuates aortic fatty streak development in rabbits," Arteriosclerosis, Thrombosis, and Vascular Biology, vol. 22, no. 7, pp. 1225-1231, 2002.

[59] J.-G. Kim, P. K. Mandal, K.-D. Choi, C.-W. Pyun, G.-E. Hong, and C.-H. Lee, "Beneficial dietary effect of turmeric and sulphur on weight gain, fat deposition and lipid profile of 
serum and liver in rats," Journal of Food Science and Technology, vol. 51, no. 4, pp. 774-779, 2014.

[60] J. A. Girón-González, C. Martínez-Sierra, C. RodriguezRamos et al., "Implication of inflammation-related cytokines in the natural history of liver cirrhosis," Liver International, vol. 24, no. 5, pp. 437-445, 2004.

[61] C. Berasain, J. Castillo, M. J. Perugorria, M. U. Latasa, J. Prieto, and M. A. Avila, "Inflammation and liver cancer," Annals of the New York Academy of Sciences, vol. 1155, no. 1, pp. 206-221, 2009.

[62] J. Prieto, "Inflammation, HCC and sex: IL-6 in the centre of the triangle," Journal of Hepatology, vol. 48, no. 2, pp. 380-381, 2008.

[63] A. Baghdasaryan, T. Claudel, A. Kosters et al., "Curcumin improves sclerosing cholangitis in Mdr2-/- mice by inhibition of cholangiocyte inflammatory response and portal myofibroblast proliferation," Gut, vol. 59, no. 4, pp. 521-530, 2010.

[64] R. Bataller and D. A. Brenner, "Liver fibrosis," Journal of Clinical Investigation, vol. 115, no. 2, pp. 209-218, 2005.

[65] S. H. Kim, H. J. Woo, Y. C. Kim, and J. H. Lee, "Inhibitory effect of curcumae longae radix on fibrogenesis in hepatic stellate cell line, LX-2," The Journal of Internal Korean Medicine, vol. 30, no. 2, pp. 306-316, 2009.

[66] E. Abdel-Lateef, F. Mahmoud, O. Hammam et al., "Bioactive chemical constituents of Curcuma longa L. rhizomes extract inhibit the growth of human hepatoma cell line (HepG2)," Acta Pharmaceutica, vol. 66, no. 3, pp. 387-398, 2016.

[67] S. Leelawat and K. Leelawat, "Molecular mechanisms of cholangiocarcinoma cell inhibition by medicinal plants," Oncology Letters, vol. 13, no. 2, pp. 961-966, 2017.

[68] P. Santiago, A. R. Scheinberg, and C. Levy, "Cholestatic liver diseases: new targets, new therapies," Therapeutic Advances in Gastroenterology, vol. 11, pp. 1-15, 2018.

[69] G. Paumgartner, "Medical treatment of cholestatic liver diseases: from pathobiology to pharmacological targets," World Journal of Gastroenterology, vol. 12, no. 28, pp. 44454454, 2006.

[70] Y. Ozaki and O. B. Liang, "Cholagogic action of the essential oil obtained from Curcuma xanthorrhiza Roxb," The Japanese Journal of Pharmacognosy, vol. 42, no. 4, pp. p257-263, 1988.

[71] Y. Wang, L. Wang, X. Zhu, D. Wang, and X. Li, "Choleretic activity of turmeric and its active ingredients," Journal of Food Science, vol. 81, no. 7, pp. 1800-1806, 2016.

[72] M.-S. Kim, S.-S. Chun, S.-H. Kim, and J.-H. Choi, "Effect of tumeric (Curcuma longa) on bile acid and UDP-glucuronyl transferase activity in rats fed a high-fat and -cholesterol diet," Journal of Life Science, vol. 22, no. 8, pp. 1064-1070, 2012.

[73] S. Li, W. Yuan, G. Deng, P. Wang, P. Yang, and B. Aggarwal, "Chemical composition and product quality control of turmeric (Curcuma longa L.)," Pharmaceutical Crops, vol. 5, no. 1 , pp. $28-54,2011$.

[74] A. Nair, A. Amalraj, J. Jacob, A. B. Kunnumakkara, and S. Gopi, "Non-curcuminoids from turmeric and their potential in cancer therapy and anticancer drug delivery formulations," Biomolecules, vol. 9, no. 1, pp. 1-36, 2019.

[75] Z. J. Dai, W. Tang, W. F. Lu et al., "Antiproliferative and apoptotic effects of $\beta$-elemene on human hepatoma HepG2 cells," Cancer Cell Iinternational, vol. 13, no. 1, pp. 1-10, 2013.

[76] F. Bao, J. Qiu, and H. Zhang, "Potential role of $\beta$-elemene on histone $\mathrm{H} 1$ in the $\mathrm{H} 22$ ascites hepatoma cell line," Molecular Medicine Reports, vol. 6, no. 1, pp. 185-190, 2012.

[77] X. Li, Z. Lin, B. Zhang et al., " $\beta$-elemene sensitizes hepatocellular carcinoma cells to oxaliplatin by preventing oxaliplatin-induced degradation of copper transporter 1," Scientific Reports, vol. 6, no. 1, pp. 1-12, 2016.

[78] J. Liu, Z. Zhang, J. Gao, J. Xie, L. Yang, and S. Hu, "Downregulation effects of beta-elemene on the levels of plasma endotoxin, serum TNF-alpha, and hepatic CD14 expression in rats with liver fibrosis," Frontiers of Medicine, vol. 5, no. 1, pp. 101-105, 2011.

[79] R. Zhu, L. Yang, L. Shen, J. Ye, J. Liu, and S. Hu, "ANG IIAT1 receptor pathway is involved in the anti-fibrotic effect of $\beta$-elemene," Journal of Huazhong University of Science and Technology [Medical Sciences], vol. 29, no. 2, pp. 177-181, 2009.

[80] J. Zheng, L. T. Ma, Q. Y. Ren et al., "The influence of astragalus polysaccharide and $\beta$-elemene on LX-2 cell growth, apoptosis and activation," BMC Gastroenterology, vol. 14, no. 224, pp. 1-6, 2014.

[81] H. Matsuda, K. Ninomiya, T. Morikawa, and M. Yoshikawa, "Inhibitory effect and action mechanism of sesquiterpenes from zedoariae rhizoma on d-galactosamine/lipopolysaccharide-induced liver injury," Bioorganic \& Medicinal Chemistry Letters, vol. 8, no. 4, pp. 339-344, 1998.

[82] T. Morikawa, H. Matsuda, K. Ninomiya, and M. Yoshikawa, "Medicinal foodstuffs. XXIX. Potent protective effects of sesquiterpenes and curcumin from zedoariae rhizoma on liver injury induced by D-galactosamine/lipopolysaccharide or tumor necrosis factor- $\alpha$," Biological \& Pharmaceutical Bulletin, vol. 25, no. 5, pp. 627-631, 2002.

[83] Y. Liu, W. Wang, B. Fang et al., "Anti-tumor effect of germacrone on human hepatoma cell lines through inducing G2/M cell cycle arrest and promoting apoptosis," European Journal of Pharmacology, vol. 698, no. 1-3, pp. 95-102, 2012.

[84] J. Hucklenbroich, R. Klein, B. Neumaier et al., "Aromaticturmerone induces neural stem cell proliferation in vitro and in vivo," Stem Cell Research \& Therapy, vol. 5, no. 4, pp. 1-9, 2014.

[85] S.-B. Cheng, L.-C. Wu, Y.-C. Hsieh et al., "Supercritical carbon dioxide extraction of aromatic turmerone from Curcuma longa Linn. induces apoptosis through reactive oxygen species-triggered intrinsic and extrinsic pathways in human hepatocellular carcinoma HepG2 cells," Journal of Agricultural and Food Chemistry, vol. 60, no. 38, pp. 9620-9630, 2012.

[86] G. G. Yue, B. C. Chan, P.-M. Hon et al., "Evaluation of in vitro anti-proliferative and immunomodulatory activities of compounds isolated from Curcuma longa," Food and Chemical Toxicology, vol. 48, no. 8-9, pp. 2011-2020, 2010.

[87] G. G.-L. Yue, H.-F. Kwok, J. K.-M. Lee et al., "Novel antiangiogenic effects of aromatic-turmerone, essential oil isolated from spice turmeric," Journal of Functional Foods, vol. 15, pp. 243-253, 2015.

[88] B. B. Aggarwal, W. Yuan, S. Li, and S. C. Gupta, "Curcumin-free turmeric exhibits anti-inflammatory and anticancer activities: identification of novel components of turmeric," Molecular Nutrition \& Food Research, vol. 57, no. 9, pp. 1529-1542, 2013.

[89] H. Dancygier, "Clinical hepatology: principles and practice of hepatobiliary diseases," Springer Science \& Business Media, 2009.

[90] B. Zhai, Y. Zeng, Z. Zeng et al., "Drug delivery systems for elemene, its main active ingredient \&beta;-elemene, and its derivatives in cancer therapy," International Journal of Nanomedicine, vol. 13, pp. 6279-6296, 2018. 